\title{
Reduction of Chern-Simons-Schrödinger Systems in One Space Dimension
}

\author{
Hyungjin Huh \\ Department of Mathematics, Chung-Ang University, Seoul 156-756, Republic of Korea \\ Correspondence should be addressed to Hyungjin Huh; huh@cau.ac.kr
}

Received 6 August 2013; Revised 31 October 2013; Accepted 31 October 2013

Academic Editor: Anjan Biswas

Copyright (C) 2013 Hyungjin Huh. This is an open access article distributed under the Creative Commons Attribution License, which permits unrestricted use, distribution, and reproduction in any medium, provided the original work is properly cited.

We study Chern-Simons-Schrödinger systems in one space dimension. We show that Chern-Simons-Schrödinger and $\mathcal{N}=2$ supersymmetric Chern-Simons-Schrödinger equations can be reduced, under the gauge condition $A_{1} \equiv 0$, to equations of $\phi$, $\psi$ only which are coupled cubic Schrödinger systems.

\section{Introduction}

In this paper, we consider the Chern-Simons-Schrödinger (CSS) system

$$
\begin{gathered}
i D_{0} \phi+D_{1} D_{1} \phi-N^{2} \phi+\frac{2}{\kappa}|\phi|^{2} \phi=0, \\
\kappa \partial_{1} N+|\phi|^{2}=0, \\
\kappa F_{01}-2 N|\phi|^{2}=0, \\
\kappa \partial_{0} N-2 \operatorname{Im}\left(\bar{\phi} D_{1} \phi\right)=0
\end{gathered}
$$

and the $\mathcal{N}=2$ supersymmetric Chern-Simons-Schrödinger (s-CSS) system

$$
\begin{gathered}
i D_{0} \phi+D_{1} D_{1} \phi-N^{2} \phi+2 \lambda_{1}|\phi|^{2} \phi+\lambda_{2}|\psi|^{2} \phi=0 \\
i D_{0} \psi+D_{1} D_{1} \psi-\psi \partial_{1} N-N^{2} \psi+\lambda_{2}|\phi|^{2} \psi=0 \\
\kappa \partial_{1} N+\left(|\phi|^{2}+|\psi|^{2}\right)=0 \\
\kappa F_{01}+\partial_{1}\left(|\psi|^{2}\right)-2 N\left(|\phi|^{2}+|\psi|^{2}\right)=0 \\
\kappa \partial_{0} N-2 \operatorname{Im}\left(\bar{\phi} D_{1} \phi\right)-2 \operatorname{Im}\left(\bar{\psi} D_{1} \psi\right)=0
\end{gathered}
$$

on $\mathbb{R}^{1+1}$. Here, $A_{0}, A_{1}$, and $N$ are real fields, $\phi, \psi$ are complex scalar fields, and $F_{01}=\partial_{0} A_{1}-\partial_{1} A_{0}$. The covariant derivative is defined by $D_{\alpha} \phi=\partial_{\alpha} \phi-i A_{\alpha} \phi,(\alpha=0,1) . \lambda_{1}, \lambda_{2}$, and $\kappa>0$ are coupling constants. The space-time derivatives of a function $f$ are denoted by $\left(\partial_{0} f, \partial_{1} f\right)=\left(\partial_{t} f, \partial_{x} f\right)$.

The CSS model was proposed in [1] to study BPS domain wall solutions. The Lagrangian density of the $(1+1)$ dimensional Chern-Simons-Schrödinger system is given by

$$
\mathscr{L}=\kappa N F_{01}+i \bar{\phi} D_{0} \phi-\left|D_{1} \phi\right|^{2}-N^{2}|\phi|^{2}+\frac{1}{\kappa}|\phi|^{4},
$$

which is obtained by the dimensional reduction of the Lagrangian density of $(2+1)$ dimensional Chern-SimonsSchrödinger system in [2]. The (s-CSS) system is derived, by the dimensional reduction, from the paper [3].

An important property of the systems CSS and s-CSS is the gauge invariance. Therefore, a solution to the systems CSS and s-CSS is formed by a class of gauge equivalent pairs $\left(\phi, A_{0}, A_{1}, N\right)$ and $\left(\phi, \psi, A_{0}, A_{1}, N\right)$, respectively. Here, we will fix the gauge by imposing the condition $A_{1} \equiv 0$. Note that temporal gauge condition $A_{0} \equiv 0$ is well known.

The motivation considering the gauge condition $A_{1} \equiv$ 0 comes from standing wave solutions of CSS. As shown in Section 2.1, the usual ansatz of standing wave leads to $A_{1} \equiv 0$. To study stability, it seems natural to study the initial value problem of CSS with the condition $A_{1} \equiv 0$. The 
other motivation is that the Schrödinger part in CSS system is written, under the Lorenz gauge condition $\partial_{0} A_{0}-\partial_{1} A_{1}=0$, as follows:

$$
\begin{aligned}
i \partial_{t} \phi & +\partial_{x} \partial_{x} \phi+A_{0} \phi-i \phi \partial_{x} A_{1} \\
& -2 i A_{1} \partial_{x} \phi-A_{1}^{2} \phi-N^{2} \phi+\frac{2}{\kappa}|\phi|^{2} \phi=0,
\end{aligned}
$$

where we have a singular derivative nonlinear term $A_{1} \partial_{x} \phi$. The gauge condition $A_{1} \equiv 0$ removes troublesome nonlinearity automatically. Note that Lorenz gauge condition was made use of in previous studies [4,5] on Maxwell-Schrödinger equations in one space dimension.

The initial value problem of the Chern-SimonsSchrödinger system in $\mathbb{R}^{2+1}$ was investigated in [6-9]. Blow-up solutions in finite time have been studied in [6] by deriving a virial identity and in [10] by the use of a pseudoconformal transformation. The existence of standing wave solutions has been studied in [11, 12]. Global energy solutions of Chern-Simons-Higgs equations in one space dimension have been studied in [13].

In this study, we consider smooth solutions which satisfy equations in the classical sense and decay properly at spatial infinity. Our first result says that CSS system can be reduced, under the gauge condition $A_{1} \equiv 0$, to the equation of $\phi$ only which is a cubic Schrödinger equation.

Theorem 1. Let one consider a smooth solution $\left(\phi, A_{0}, N\right)$ of (15)-(18) satisfying $\phi \in C\left([0, T] ; H^{2}(\mathbb{R})\right)$. Then, the scalar field $\phi$ is also a solution to the following Schrödinger equation:

$$
i \partial_{t} \phi+\partial_{x} \partial_{x} \phi+\frac{2}{\kappa}|\phi|^{2} \phi=0 .
$$

The s-CSS system can be reduced, under the gauge condition $A_{1} \equiv 0$, to the system of $\phi$ and $\psi$ only.

Theorem 2. Let one consider a smooth solution $\left(\phi, \psi, A_{0}, N\right)$ of the system (27)-(31) satisfying $\phi, \psi \in C\left([0, T] ; H^{2}(\mathbb{R})\right)$. Then, the scalar fields $\phi$ and $\psi$ are also a solution to the following coupled Schrödinger equations:

$$
\begin{aligned}
& i \partial_{t} \phi+\partial_{x} \partial_{x} \phi+2 \lambda_{1}|\phi|^{2} \phi+\left(\lambda_{2}+\frac{1}{\kappa}\right)|\psi|^{2} \phi=0, \\
& i \partial_{t} \psi+\partial_{x} \partial_{x} \psi+\frac{2}{\kappa}|\psi|^{2} \psi+\left(\lambda_{2}+\frac{1}{\kappa}\right)|\phi|^{2} \psi=0 .
\end{aligned}
$$

Remark 3. (i) The model (5) is a cubic Schrödinger equation with attractive potential, and the system (6) is the two coupled Schrödinger equations. In particular, when $\lambda_{2}=-1 / \kappa$, the equations are two versions of a single nonlinear Schrödinger equation which is integrable.

(ii) Looking for standing wave solutions of $(6), \phi(x, t)=$ $e^{i \omega_{1} t} u(x)$ and $\psi(x, t)=e^{i \omega_{2} t} v(x)$, one can check that $u$ and $v$ satisfy the following system:

$$
\begin{gathered}
\partial_{x} \partial_{x} u-\omega_{1} u+2 \lambda_{1}|u|^{2} u+\left(\lambda_{2}+\frac{1}{\kappa}\right)|v|^{2} u=0, \\
\partial_{x} \partial_{x} v-\omega_{2} v+\frac{2}{\kappa}|v|^{2} v+\left(\lambda_{2}+\frac{1}{\kappa}\right)|u|^{2} v=0 .
\end{gathered}
$$

The existence of standing waves and their properties have been studied extensively, for instance, in [14-16].

Theorem 1 is proved in Section 2, and Theorem 2 is proved in Section 3. We give concluding remark in Section 4. We use the standard Sobolev space $H^{2}(\mathbb{R})$ which denotes the set of weakly differentiable functions $u$ on $\mathbb{R}$ such that $u, \partial_{x} u$, and $\partial_{x} \partial_{x} u$ are square integrable.

\section{Reduction of \\ Chern-Simons-Schrödinger System}

Here, we consider the reduction of Chern-SimonsSchrödinger system in one space dimension. In Section 2.1, we investigate standing wave solutions of CSS system, and Theorem 1 is proved in Section 2.2.

2.1. Standing Wave Solutions of CSS System. In this section, we look for standing wave solutions of the form

$$
\begin{array}{cc}
\phi(t, x)=e^{i \omega t} u(x), & A_{0}(t, x)=A_{0}(x), \\
A_{1}(t, x)=A_{1}(x), & N(t, x)=N(x),
\end{array}
$$

where $\omega$ is a real constant and $u$ is a real-valued function. The fourth equation in (1) leads us to $A_{1} \equiv 0$. Then, we have from (1) the following:

$$
\begin{gathered}
u^{\prime \prime}-\omega u+A_{0} u-N^{2} u+\frac{2}{\kappa}|u|^{2} u=0, \\
\kappa N^{\prime}+u^{2}=0, \\
\kappa A_{0}^{\prime}+2 N u^{2}=0,
\end{gathered}
$$

where ' denotes a derivative $d / d x$. From (10) and (11), we may have the following expressions, with a boundary condition $N(-\infty)=0=A_{0}(-\infty)$ :

$$
\begin{aligned}
& N(x)=-\frac{1}{\kappa} \int_{-\infty}^{x}|u(y)|^{2} d y, \\
& A_{0}(x)=-\frac{2}{\kappa} \int_{-\infty}^{x} N(y)|u(y)|^{2} d y .
\end{aligned}
$$

A simple calculation shows that $(d / d x)\left(A_{0}-N^{2}\right)=0$ which implies $\left(A_{0}-N^{2}\right)(x)=\left(A_{0}-N^{2}\right)(-\infty)=0$. Then, (9) becomes

$$
u^{\prime \prime}-\omega u+\frac{2}{\kappa}|u|^{2} u=0
$$

We may obtain a solution $u(x)=\sqrt{\kappa \omega} \operatorname{sech}(\sqrt{\omega} x)$ and $N(x)=$ $-\sqrt{\omega}(1+\tanh (\sqrt{\omega} x))$.

2.2. Reduction of CSS System. The (CSS) system (1) is invariant under the following gauge transformation:

$$
\phi \longrightarrow \phi e^{i \chi}, \quad A_{\alpha} \longrightarrow A_{\alpha}+\partial_{\alpha} \chi, \quad N \longrightarrow N
$$


where $\chi: \mathbb{R}^{1+1} \rightarrow \mathbb{R}$ is a smooth function. Here, we impose the gauge condition $A_{1} \equiv 0$ which reformulates the CSS system (1) as follows:

$$
\begin{gathered}
i \partial_{t} \phi+A_{0} \phi+\partial_{x} \partial_{x} \phi-N^{2} \phi+\frac{2}{\kappa}|\phi|^{2} \phi=0 \\
\kappa \partial_{x} N+|\phi|^{2}=0 \\
\kappa \partial_{x} A_{0}+2 N|\phi|^{2}=0 \\
\kappa \partial_{t} N-2 \operatorname{Im}\left(\bar{\phi} \partial_{x} \phi\right)=0 .
\end{gathered}
$$

From (16) and (17), we have, with a boundary condition $N(-\infty)=0=A_{0}(-\infty)$, the following representations:

$$
\begin{aligned}
& N(x, t)=-\frac{1}{\kappa} \int_{-\infty}^{x}|\phi(y, t)|^{2} d y, \\
& A_{0}(x, t)=-\frac{2}{\kappa} \int_{-\infty}^{x} N(y, t)|\phi(y, t)|^{2} d y .
\end{aligned}
$$

Let us check the compatibility of (18) with other (15)-(17). Multiplying (15) by $\bar{\phi}$ and taking imaginary part, we have

$$
\partial_{t}|\phi|^{2}+2 \operatorname{Im}\left(\bar{\phi} \partial_{x} \partial_{x} \phi\right)=0
$$

Taking time derivative of $N$ in (19) and considering (20), we have

$$
\begin{aligned}
\kappa \partial_{t} N(x, t) & =-\int_{-\infty}^{x} \partial_{t}|\phi|^{2}(y, t) d y \\
& =\int_{-\infty}^{x} 2 \partial_{y} \operatorname{Im}\left(\bar{\phi} \partial_{y} \phi\right)(y, t) d y \\
& =2 \operatorname{Im}\left(\bar{\phi} \partial_{x} \phi\right),
\end{aligned}
$$

where $\operatorname{Im}\left(\bar{\phi} \partial_{x} \partial_{x} \phi\right)=\partial_{x} \operatorname{Im}\left(\bar{\phi} \partial_{x} \phi\right)$ and $\phi(\cdot, t) \in H^{2}(\mathbb{R})$ are used.

We have showed that the study of (15)-(18) reduces to the following system:

$$
i \partial_{t} \phi+A_{0} \phi+\partial_{x} \partial_{x} \phi-N^{2} \phi+\frac{2}{\kappa}|\phi|^{2} \phi=0
$$

where $N$ and $A_{0}$ are defined by (19). Moreover, we can check that, using (19):

$$
\kappa \partial_{x}\left(A_{0}-N^{2}\right)=-2 N|\phi|^{2}-2 N\left(-|\phi|^{2}\right)=0,
$$

which implies $\left(A_{0}-N^{2}\right)(x, t)=\left(A_{0}-N^{2}\right)(-\infty, t)=0$. Therefore, (22) reduces finally to

$$
i \partial_{t} \phi+\partial_{x} \partial_{x} \phi+\frac{2}{\kappa}|\phi|^{2} \phi=0
$$

which proves Theorem 1 .

\section{Reduction of s-CSS System}

The Lagrangian density of the $(1+1)$ dimensional $\mathcal{N}=2$ supersymmetric Chern-Simons-Schrödinger system is given by

$$
\begin{aligned}
\mathscr{L} & =\kappa N F_{01}+i \bar{\phi} D_{0} \phi-\left|D_{1} \phi\right|^{2}+i \bar{\psi} D_{0} \psi-\left|D_{1} \psi\right|^{2} \\
& -|\psi|^{2} \partial_{1} N-N^{2}\left(|\phi|^{2}+|\psi|^{2}\right)+\lambda_{1}|\phi|^{4}+\lambda_{2}|\phi|^{2}|\psi|^{2},
\end{aligned}
$$

which is obtained by the dimensional reduction of the Lagrangian density of $(2+1)$ dimensional $\mathcal{N}=2$ supersymmetric Chern-Simons-Schrödinger system in [3]. The s-CSS system (2) is invariant under the following gauge transformation:

$$
\begin{gathered}
\phi \longrightarrow \phi e^{i \chi}, \quad \psi \longrightarrow \psi e^{i \chi}, \\
A_{\alpha} \longrightarrow A_{\alpha}+\partial_{\alpha} \chi, \quad N \longrightarrow N,
\end{gathered}
$$

where $\chi: \mathbb{R}^{1+1} \rightarrow \mathbb{R}$ is a smooth function. We consider the gauge condition $A_{1} \equiv 0$ which reformulates the s-CSS system (2) as follows:

$$
\begin{gathered}
i \partial_{t} \phi+A_{0} \phi+\partial_{x} \partial_{x} \phi-N^{2} \phi+2 \lambda_{1}|\phi|^{2} \phi+\lambda_{2}|\psi|^{2} \phi=0 \\
i \partial_{t} \psi+A_{0} \psi+\partial_{x} \partial_{x} \psi-\psi \partial_{x} N-N^{2} \psi+\lambda_{2}|\phi|^{2} \psi=0 \\
\kappa \partial_{x} N+\left(|\phi|^{2}+|\psi|^{2}\right)=0 \\
\kappa \partial_{x} A_{0}-\partial_{x}\left(|\psi|^{2}\right)+2 N\left(|\phi|^{2}+|\psi|^{2}\right)=0 \\
\kappa \partial_{t} N-2 \operatorname{Im}\left(\bar{\phi} \partial_{x} \phi\right)-2 \operatorname{Im}\left(\bar{\psi} \partial_{x} \psi\right)=0 .
\end{gathered}
$$

From (29) and (30), we have, with a boundary condition $N(-\infty)=0=A_{0}(-\infty)$, the following representations:

$$
\begin{aligned}
& \kappa N(x, t)=-\int_{-\infty}^{x}\left(|\phi|^{2}+|\psi|^{2}\right)(y, t) d y, \\
& \kappa A_{0}(x, t)=|\psi(x, t)|^{2}-2 \int_{-\infty}^{x} N\left(|\phi|^{2}+|\psi|^{2}\right)(y, t) d y .
\end{aligned}
$$

Let us check the compatibility of (31) with other (27)-(30). Multiplying (27) and (28) by $\bar{\phi}$ and $\bar{\psi}$, respectively, and taking imaginary part, we have

$$
\begin{aligned}
& \partial_{t}|\phi|^{2}+2 \operatorname{Im}\left(\bar{\phi} \partial_{x} \partial_{x} \phi\right)=0, \\
& \partial_{t}|\psi|^{2}+2 \operatorname{Im}\left(\bar{\psi} \partial_{x} \partial_{x} \psi\right)=0 .
\end{aligned}
$$

Taking time derivative of $N$ in (32) and considering (33), we have

$$
\begin{aligned}
\kappa \partial_{t} N(x, t)= & -\int_{-\infty}^{x}\left(\partial_{t}|\phi|^{2}+\partial_{t}|\psi|^{2}\right)(y, t) d y \\
= & \int_{-\infty}^{x}\left(2 \partial_{y} \operatorname{Im}\left(\bar{\phi} \partial_{y} \phi\right)+2 \partial_{y} \operatorname{Im}\left(\bar{\psi} \partial_{y} \psi\right)\right) \\
& \times(y, t) d y \\
= & 2 \operatorname{Im}\left(\bar{\phi} \partial_{x} \phi\right)+2 \operatorname{Im}\left(\bar{\psi} \partial_{x} \psi\right) .
\end{aligned}
$$


We have showed that the study of (27)-(31) reduces to the following system:

$$
\begin{aligned}
& i \partial_{t} \phi+A_{0} \phi+\partial_{x} \partial_{x} \phi-N^{2} \phi+2 \lambda_{1}|\phi|^{2} \phi+\lambda_{2}|\psi|^{2} \phi=0, \\
& i \partial_{t} \psi+A_{0} \psi+\partial_{x} \partial_{x} \psi-\psi \partial_{x} N-N^{2} \psi+\lambda_{2}|\phi|^{2} \psi=0,
\end{aligned}
$$

where $N$ and $A_{0}$ are defined by (32). Now we can check that $\partial_{x}\left(\kappa A_{0}-\kappa N^{2}-|\psi|^{2}\right)=0$, which implies

$$
\left(\kappa A_{0}-\kappa N^{2}-|\psi|^{2}\right)(x, t)=0 .
$$

Taking (32) and (36) into account, we can check that

$$
\left(A_{0}-\partial_{x} N-N^{2}\right)(x, t)=\frac{1}{\kappa}\left(|\phi|^{2}+2|\psi|^{2}\right)(x, t) .
$$

Then, considering (36) and (37), the system (35) reduces to

$$
\begin{aligned}
& i \partial_{t} \phi+\partial_{x} \partial_{x} \phi+2 \lambda_{1}|\phi|^{2} \phi+\left(\lambda_{2}+\frac{1}{\kappa}\right)|\psi|^{2} \phi=0, \\
& i \partial_{t} \psi+\partial_{x} \partial_{x} \psi+\frac{2}{\kappa}|\psi|^{2} \psi+\left(\lambda_{2}+\frac{1}{\kappa}\right)|\phi|^{2} \psi=0,
\end{aligned}
$$

which proves Theorem 2 .

\section{Concluding Remark}

As we pointed out in Section 1, Schrödinger equations with electromagnetic field like Maxwell-Schrödinger and Chern-Simons-Schrödinger have singular derivative nonlinear terms which give difficulties in analysis of the PDEs. Those challenging problems have prompted development of analytic methods and the results [4-12,17] regarding issues such as existence, blowup, and asymptotic behaviors of the solution. In this aspect, the results of this study seem interesting and quite unique. CSS and s-CSS systems with gauge condition $A_{1} \equiv 0$ reduce to coupled cubic Schrödinger equations which are much easier from analytic and numerical point of view. We could not obtain similar reduction result for the related equations like Chern-Simons-Higgs and ChernSimons-Dirac in one space dimension which have their own interesting structures $[13,18]$.

\section{Conflict of Interests}

The author declares that there is no conflict of interests regarding the publication of this paper.

\section{Acknowledgment}

The author was supported by the Basic Science Research Program through the National Research Foundation of Korea (NRF) funded by the Ministry of Education, Science and Technology (2011-0015866).

\section{References}

[1] H. c. Kao, K. Lee, and T. Lee, "BPS domain wall solutions in selfdual Chern-Simons-Higgs systems," Physical Review D, vol. 55, no. 10, pp. 6447-6453, 1997.
[2] R. Jackiw and S. Y. Pi, "Classical and quantal nonrelativistic Chern-Simons theory," Physical Review D, vol. 42, no. 10, pp. 3500-3513, 1990.

[3] M. Leblanc, G. Lozano, and H. Min, "Extended superconformal Galilean symmetry in Chern-Simons matter systems," Annals of Physics, vol. 219, no. 2, pp. 328-348, 1992.

[4] K. Nakamitsu and M. Tsutsumi, "The Cauchy problem for the coupled Maxwell-Schrödinger equations," Journal of Mathematical Physics, vol. 27, no. 1, pp. 211-216, 1986.

[5] Y. Tsutsumi, "Global existence and uniqueness of energy solutions for the Maxwell-Schrödinger equations in one space dimension," Hokkaido Mathematical Journal, vol. 24, no. 3, pp. 617-639, 1995.

[6] L. Bergé, A. de Bouard, and J. C. Saut, "Blowing up timedependent solutions of the planar, Chern-Simons gauged nonlinear Schrödinger equation," Nonlinearity, vol. 8, no. 2, pp. 235253,1995

[7] S. Demoulini, "Global existence for a nonlinear SchroedingerChern-Simons system on a surface," Analyse Non Linéaire, vol. 24, no. 2, pp. 207-225, 2007.

[8] S. Demoulini and D. Stuart, "Adiabatic limit and the slow motion of vortices in a Chern-Simons-Schrödinger system," Communications in Mathematical Physics, vol. 290, no. 2, pp. 597-632, 2009.

[9] H. Huh, "Energy solution to the Chern-Simons-Schrödinger equations," Abstract and Applied Analysis, vol. 2013, Article ID 590653, 7 pages, 2013.

[10] H. Huh, "Blow-up solutions of the Chern-Simons-Schrödinger equations," Nonlinearity, vol. 22, no. 5, pp. 967-974, 2009.

[11] J. Byeon, H. Huh, and J. Seok, "Standing waves of nonlinear Schrödinger equations with the gauge field," Journal of Functional Analysis, vol. 263, no. 6, pp. 1575-1608, 2012.

[12] H. Huh, "Standing waves of the Schrödinger equation coupled with the Chern-Simons gauge field," Journal of Mathematical Physics, vol. 53, no. 6, Article ID 063702, 8 pages, 2012.

[13] H. Huh, "Global energy solutions of Chern-Simons-Higgs equations in one space dimension," Preprint.

[14] D. Cao, I. L. Chern, and J. C. Wei, "On ground state of spinor Bose-Einstein condensates," Nonlinear Differential Equations and Applications, vol. 18, no. 4, pp. 427-445, 2011.

[15] S. V. Manakov, "On the theory of two-dimensional stationary self-focusing of electromagnetic waves," Journal of Experimental and Theoretical Physics, vol. 38, pp. 248-253, 1974.

[16] J. Yang, "Classification of the solitary waves in coupled nonlinear Schrödinger equations," Physica D, vol. 108, no. 1-2, pp. $92-$ 112, 1997.

[17] T. Wada, "Smoothing effects for Schrödinger equations with electro-magnetic potentials and applications to the MaxwellSchrödinger equations," Journal of Functional Analysis, vol. 263, no. 1, pp. 1-24, 2012.

[18] H. Huh, "Remarks on Chern-Simons-Dirac equations in one space dimension," Preprint. 


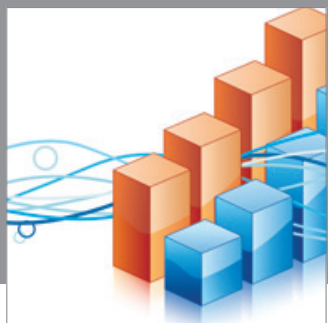

Advances in

Operations Research

mansans

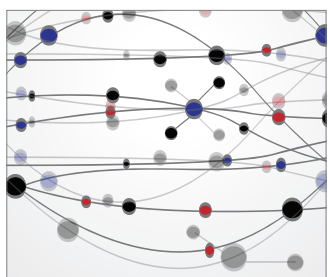

The Scientific World Journal
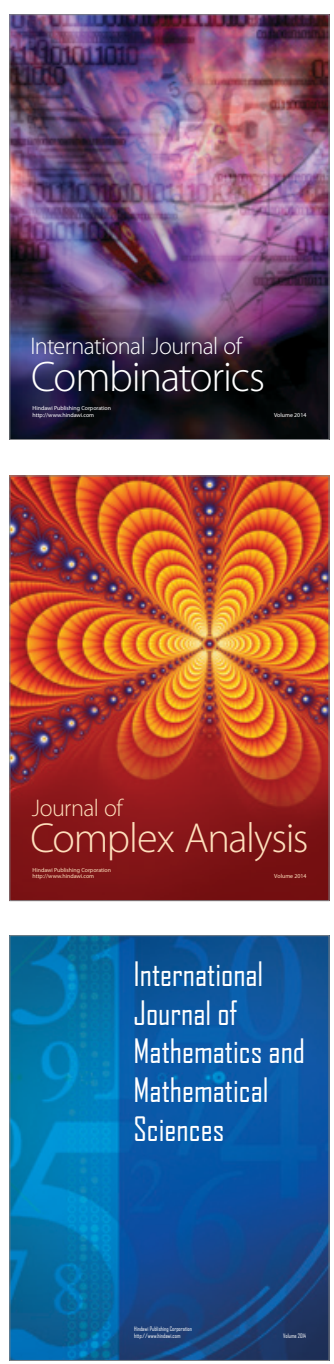
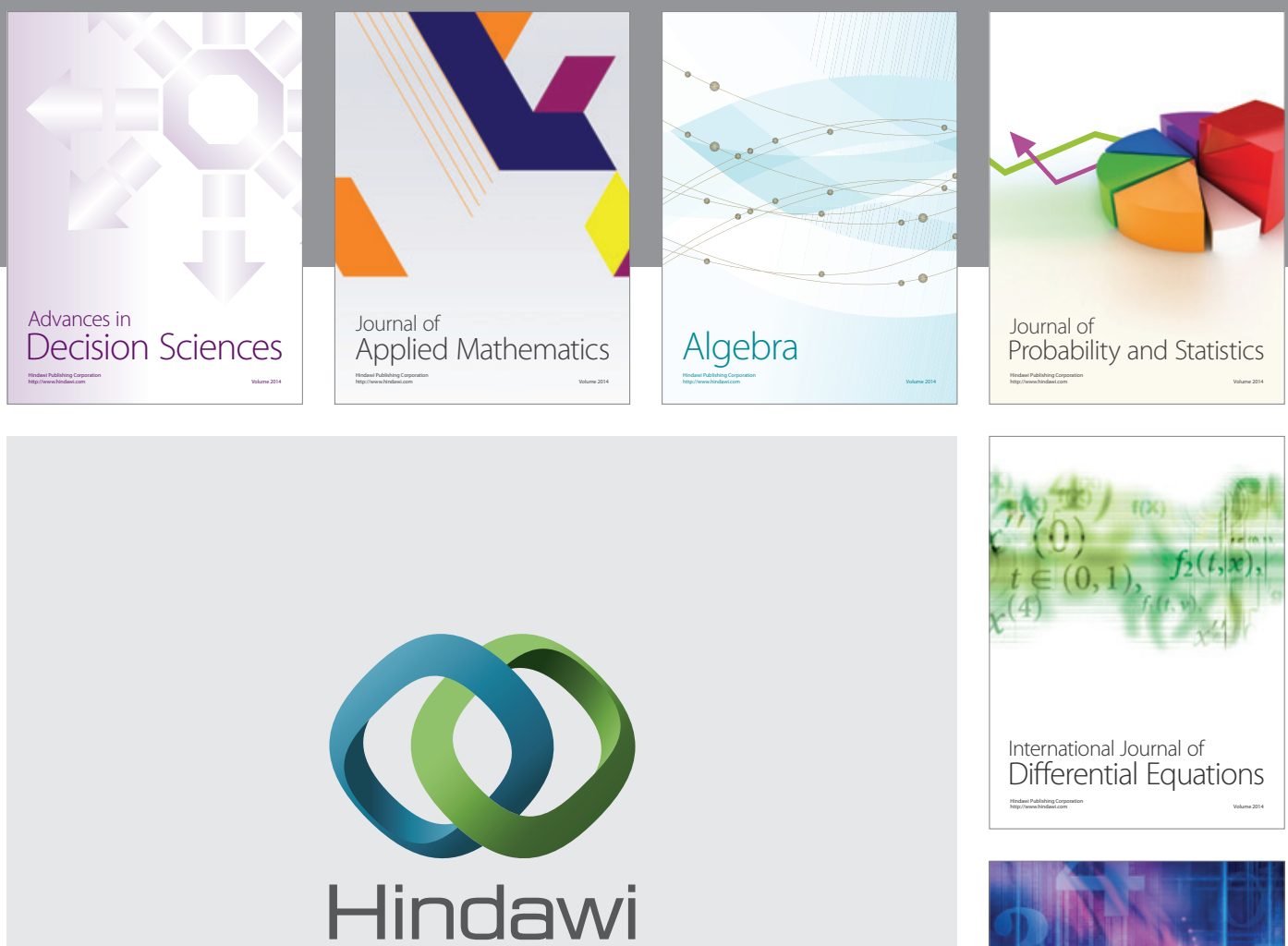

Submit your manuscripts at http://www.hindawi.com
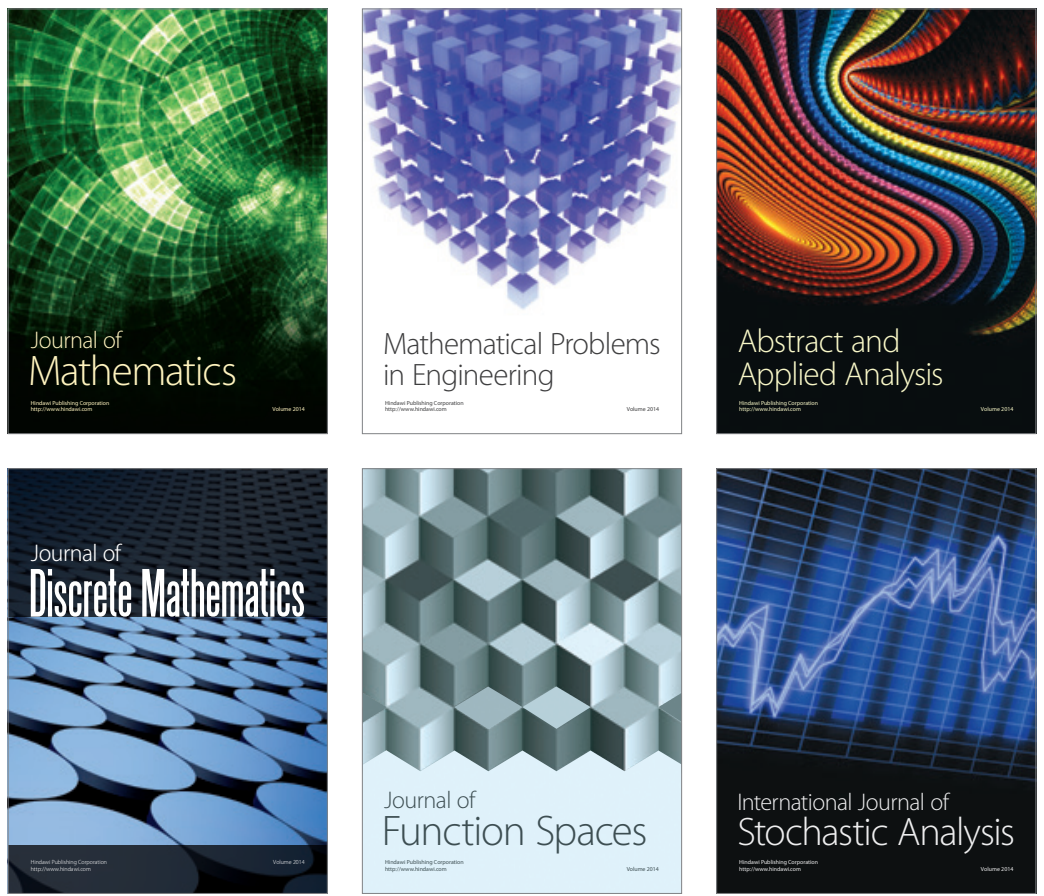

Journal of

Function Spaces

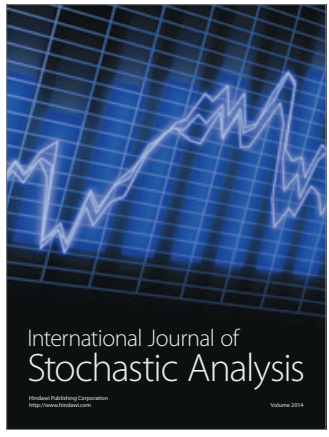

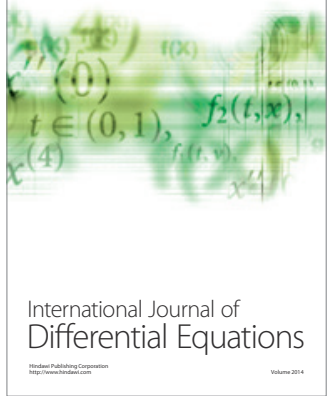
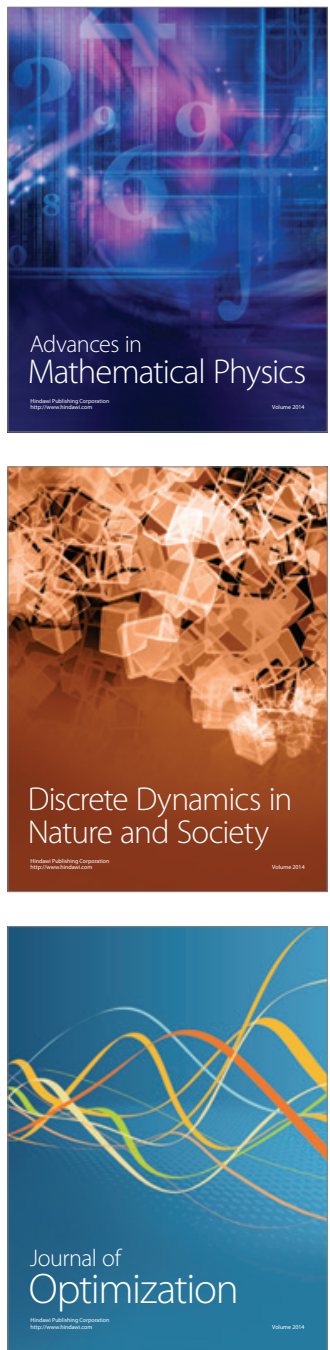